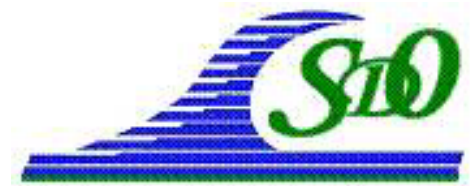

XI ${ }^{\text {èmes }}$ Journées Nationales Génie Côtier - Génie Civil

Les Sables d'Olonne, 22-25 juin 2010

DOI:10.5150/jngcgc.2010.047-R C Editions Paralia CFL

disponible en ligne - http://www.paralia.fr - available online

\title{
Etude des processus d'envasement du port de St-Jean-de Luz/Ciboure via une approche empirique
}

\author{
Didier RIHOUEY ${ }^{1}$
}

1. CASAGEC, UFR Sciences et Techniques de la côte basque,

1 Allée du Parc Montaury, 64600 Anglet, France.

didier.rihouey@univ-pau.fr

\section{Résumé :}

Le port de Saint-Jean-de-Luz/Ciboure, situé au débouché de l'estuaire de la Nivelle, présente une tendance à l'envasement plus importante ces dernières années. Ce phénomène constitue une problématique majeure pour le gestionnaire du port qui s'est vu obligé d'augmenter la fréquence des dragages pour garantir l'accès des navires de pêche. Le développement d'outils prévisionnels d'évolution bathymétrique constitue donc une problématique majeure afin d'optimiser ces dragages coûteux. Cette étude présente un modèle morphodynamique empirique, dans lequel les évolutions tridimensionnelles de la bathymétrie sont prises en compte par analyse factorielle et reliées linéairement aux forçages de débit et de dragage. Ainsi, la forte corrélation entre observations et prédictions du modèle empirique permet de valider les hypothèses réalisées sur les valeurs seuils du débit de la Nivelle et met en évidence le lien entre la tendance renforcée à l'envasement du port et l'augmentation de l'intensité des épisodes de crues depuis 2001.

\section{Mots-clés :}

Envasements portuaire - Dragage - Analyse factorielle de données bathymétriques Modèle empirique

\section{Introduction}

Préserver une bonne accessibilité aux installations portuaires reste l'un des problèmes majeurs qui se pose aux gestionnaires des ports. L'objectif principal est de maintenir un chenal de navigation suffisamment profond pour permettre aux navires d'accéder aux quais. L'amélioration des connaissances et le développement d'outils prévisionnels d'évolution bathymétrique sont donc devenus des problématiques majeures et stratégiques en termes de gestion des activités de dragage. On distingue deux grandes catégories de modèles d'évolution morphodynamique : les modèles numériques basés sur les équations fondamentales de la dynamique, les modèles empiriques basés sur l'analyse statistique de données in situ de morphologie et des forçages. Ce dernier type de modèle, appliqué à l'envasement du port de Saint-Jean-de-Luz/Ciboure, constitue l'objet de cet article. Les modèles empiriques sont déjà largement utilisés par les météorologues et les océanographes, qui disposent d'un grand nombre de données in 
situ par le biais des stations d'observation et de la télédétection. Depuis les années 80 , les modèles empiriques basés sur l'Analyse Factorielle (AFC) de données topobathymétriques couplée à un modèle linéaire se sont révélés efficaces pour décrire les évolutions morphologiques de plages (AUBREY et al., 1980; HSU et al., 1994 ; RIHOUEY, 2004). Plus récemment, le spectre d'application de cette technique c'est élargi aux "grands" bancs de sable et aux embouchures de rivières (REEVE et al., 2008 ; RIHOUEY \& DUBRANNA, 2008). Cette étude présente un modèle morphodynamique empirique, dans lequel les évolutions tridimensionnelles de la bathymétrie sont prises en compte par AFC et reliées ensuite aux forçages climatiques et anthropiques via une régression linéaire. Le principe de l'AFC et la formulation utilisée pour relier les évolutions bathymétriques aux forçages climatiques et anthropiques, sont présentées dans la section 2. Le site d'étude et les données utilisées dans le modèle sont ensuite détaillés dans la section 3. Enfin, les résultats du modèle, ses limitations et les perspectives sont analysées et discutées dans cette section 4 .

\section{Formulation du modèle empirique}

Le principe de l'AFC appliquée à une surface, consiste à exprimer la profondeur $h\left(\xi_{l}, t_{k}\right)$ par des combinaisons linéaires de produits de fonctions (ou modes) spatiales et temporelles. Considérons un échantillon de $K$ surfaces du fond comportant chacune $L$ profondeurs $h\left(\xi_{l}, t_{k}\right)$, où les $\xi_{l}$ représentent les points sur une grille régulière, avec $1 \leq l \leq L$ et $1 \leq k \leq K$. Ainsi, l'objectif de l'AFC est d'exprimer les données sous la forme :

$$
h\left(\xi_{l}, t_{k}\right)=\sum_{p=1}^{L} C_{p}\left(t_{k}\right) \cdot e_{p}\left(\xi_{l}\right)
$$

où $e_{p}$ sont les vecteurs propres de la matrice des corrélations $A$ et $C_{p}$ les modes temporels correspondant. $A$ est une matrice symétrique réelle. Elle est donc diagonalisable dans $\Re$, possède $L$ valeurs propres $\lambda_{p}$ avec $1 \leq p \leq L$ et les vecteurs propres correspondant aux valeurs propres $\lambda_{p}$ satisfont l'équation matricielle :

$$
A e_{p}=\lambda_{p} e_{p}
$$

Un résultat important de cette diagonalisation est que la somme des valeurs propres est égale à la trace de la matrice $A$. Ainsi, chaque valeur propre représente un certain pourcentage de l'inertie des données initiales. De plus, si $\lambda_{1}$ et $e_{1}\left(\xi_{l}\right)$ sont respectivement la plus grande valeur propre et son vecteur propre associé, la fonction $e_{1}\left(\xi_{l}\right) \sqrt{\lambda_{1}}$ représente la bathymétrie moyenne du port. Généralement, les 2 ou 3 premiers modes représentent plus de $99 \%$ des données initiales et suffisent pour décrire l'évolution de la bathymétrie. Le premier mode spatial représente la bathymétrie moyenne au cours de la période d'étude. Les modes d'ordres supérieurs représentent quant à eux les variations par rapport à la morphologie moyenne et sont liés aux zones où les variations bathymétriques sont les plus importantes. L'évolution d'un estuaire est 
dépendante de l'état antérieur de sa morphologie et des forçages hydro-météorologiques et/ou anthropiques. Ces forçages possédant un caractère stochastique, l'évolution morphodynamique d'un estuaire peut être décrite comme un processus markovien, défini comme une succession d'états morphologiques interdépendants (SONU \& JAMES, 1973). Un modèle simple consiste à relier linéairement les données initiales et les données que l'on cherche à prédire de la façon suivante :

$P=B D$

Où : $P$ est une matrice $n \times N$ des $n$ "quantités" à prédire; $D$ est une matrice $m \times N$ des $m$ paramètres des variables; $B$ est une matrice des coefficients $n \times m ; N$ est le nombre d'observations de ces "quantités" utilisées pour la prédiction. Les coefficients de $B$ sont donnés par la régression linéaire :

$$
B=C_{P D} C_{D D^{-1}}=\left(P D^{T}\right)\left(D D^{T}\right)^{-1}
$$

Où : $C_{P D}$ est la matrice des covariances entre les données à prédire et les données initiales ; $C_{D D}$ est la matrice d'auto covariances des données initiales. La matrice $B$ peut être utilisée pour prédire les variables utilisées dans la régression (hindcast) ou pour prédire d'autres variables (forecast) non utilisées dans la régression. Si l'on note $\hat{P}$ la matrice des variables prédites à l'aide de la matrice $B$, l'équation 4 s'exprime alors par :

$$
\hat{P}=B D
$$

Les forçages à prendre en compte dans un modèle empirique dépendent du type de système côtier étudié et de l'échantillonnage temporel des données. (AUBREY et al., 1980) et (HSU et al., 1986; HSU et al., 1994) ont appliqué ce modèle linéaire en utilisant les modes temporels $C_{2}(t), C_{3}(t), \ldots, C_{n}(t)$ respectivement issus d'AFC 1D et 2D de leurs données bathymétriques de plages, couplés à des forçages de houle. Au regard des caractéristiques de la Nivelle (Section 3.2) et du jeu de données disponibles (Section 3.2), le modèle présenté ici prend en compte le débit du fleuve $(Q)$, et les activités de dragage $(D r)$ comme forçages. En utilisant le même principe avec les modes temporels $C_{1}(t), C_{2}(t)$, l'équation 5 devient :

$$
\left[\begin{array}{c}
\hat{C}_{1}(t+1) \\
\hat{C}_{2}(t+1)
\end{array}\right]=\left[\begin{array}{llll}
b_{11} & b_{12} & b_{13} & b_{14} \\
b_{21} & b_{22} & b_{23} & b_{24}
\end{array}\right]\left[\begin{array}{c}
\hat{C}_{1}(t) \\
\hat{C}_{2}(t) \\
Q(t \rightarrow t+1) \\
D r(t \rightarrow t+1)
\end{array}\right]
$$

Où : $\hat{C}_{1}(t)$ et $\hat{C}_{2}(t)$ sont les modes temporels prédits ; $b_{11}, b_{12}, \ldots, b_{34}$ sont les coefficients déterminés par l'équation $4 ; Q$ et $D r$ sont les paramètres de forçages considérés entre les temps $t$ et $t+1$. Le calcul itératif de l'équation 6 permet alors de prédire l'évolution du premier et du second mode temporel. 


\section{Présentation du site d'étude et des données utilisées dans le modèle}

\subsection{Site d'étude}

Le port de Saint-Jean-de-Luz/Ciboure est situé en fond de baie au débouché de l'estuaire de la Nivelle, petit fleuve parcourant $39 \mathrm{~km}$ avant d'arriver dans le Golfe de Gascogne sur le littoral Basque. Cette configuration, protège l'estuaire de l'action des houles et la Nivelle peut être caractérisée comme un estuaire dominé par la marée (de type mésotidal semi-diurne de période de $12 \mathrm{~h} 25^{\prime}$ '). Au droit de la baie de Saint-Jean-deLuz/Ciboure, l'amplitude moyenne de la marée est de $3.85 \mathrm{~m}$ en vives-eaux et de $1.85 \mathrm{~m}$ en mortes-eaux.

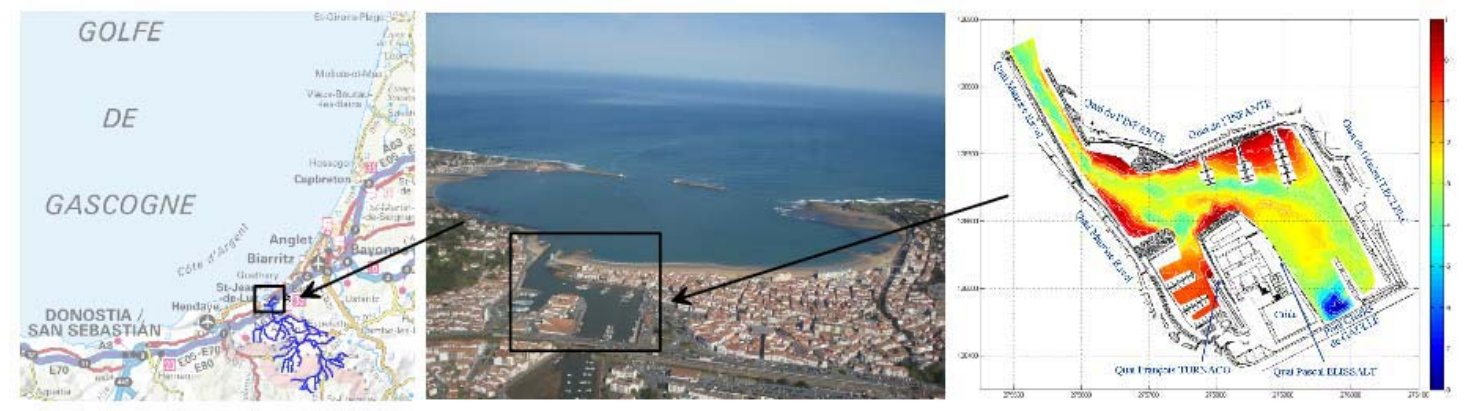

Figure 1. Situation géographique du port de Saint-Jean-de-Luz/Ciboure.

Le bassin versant de la Nivelle, qui s'étend sur $238 \mathrm{~km}^{2}$ peut être différencié en deux parties distinctes : une zone amont, caractérisée par une forte pente $(3,8 \%)$ qui engendre un régime torrentiel, et une zone aval, moins encaissée et qui possède une pente moins accentuée $(0,11 \%)$. Ce bassin versant est soumis à un climat océanique tempéré humide avec une pluviométrie moyenne de $1700 \mathrm{~mm} \mathrm{an}^{-1}$ à Saint-Pée-sur-Nivelle (MANEUX et al., 1999). La Nivelle possède un débit moyen de $5 \mathrm{~m}^{3} \mathrm{~s}^{-1}$, un débit biennal de l'ordre de $50 \mathrm{~m}^{3} \mathrm{~s}^{-1}$ et un débit décennal de l'ordre de $80 \mathrm{~m}^{3} \mathrm{~s}^{-1}$ (http://www.hydro.eaufrance.fr). L'addition de la forte pluviométrie, des pentes fortes amont et de la géologie du bassin versant font de la Nivelle une des zones des plus érosives de France (CHARRITTON, 1996 ; COYNEL et al., 2005). La concentration moyenne en Matière En Suspension (MES) est d'environ $80 \mathrm{mg} \mathrm{L}^{-1}$, ceci induit un flux de MES de l'ordre de $11 \times 10^{3} \mathrm{tan}^{-1}$ soit, une charge spécifique annuelle de l'ordre de $75 \mathrm{t} \mathrm{km}^{-2} \mathrm{an}^{-1}$ (COYNEL et al., 2005). Ces apports de matière sont à l'origine de l'envasement du port qui s'est intensifié ces dernières années. En effet, les échouages répétés des navires de pêche ont obligé le Conseil général des Pyrénées Atlantiques (en charge d'entretenir le patrimoine portuaire pèche et plaisance) à procéder au dragage du port en 2002, 2005, 2007 alors que la fréquence de dragage était auparavant d'une dizaine d'années. Notons que les analyses granulométriques $(80 \%$ des matériaux ont un diamètre inférieur à $67 \mu \mathrm{m})$ réalisées au sein du port (CREOCEAN, 2001) confirment l'origine terrestre des matériaux dragués. 


\subsection{Données utilisées}

Deux sources de données bathymétriques sont utilisées. La première, mise à disposition par le CG64 couvre une période de 15 ans (de 1991 à 2006) et se présentent sous la forme de cartes papiers, de fichiers DXF ou de nuages de point xyz en format txt. Notons que l'espacement temporel des levés est de deux ans de 1991 à 1997 et de un an de 1998 à 2007. De plus, la densité de point et la couverture spatiale des mesures est très fluctuante d'un levé à l'autre. La seconde source de donnée est issue d'un suivi bathymétrique trimestrielle réalisée par l'équipe de CASAGEC depuis octobre 2007.

En se qui concerne les débits de la Nivelle, ils sont mesurés depuis 1969 à la station limnimétrique de Cherchebruit (gérée par la DIREN Aquitaine et le Service de Prévision des Crues (SPC) du Bassin de l'Adour). Le choix du forçage de débit a été réalisé de façon à représenter au mieux les quantités de MES susceptibles de venir sédimenter dans le port. Selon la bibliographie (CHARRITTON, 1996; MANEUX et al., 1999 ; COYNEL et al., 2005) les crues véhiculent l'essentiel des transferts de MES. Il reste alors à définir une valeur critique de débit à partir de laquelle on observe un transport de MES susceptible de venir sédimenter dans le port. Or au cours du suivi bathymétrique de l'année 2008, aucun envasement notoire n'a été observé au sein du port. Le débit de la Nivelle n'ayant pas excédé $45 \mathrm{~m}^{3} \mathrm{~s}^{-1}$, cette valeur a été choisie comme valeur "seuil". De plus, afin que les crues les plus extrêmes aient un impact plus important sur la sédimentation du port, différents types de crue ont été utilisés comme forçages dans le modèle : les crues biennales correspondant à $4 \mathrm{~m}^{3} \mathrm{~s}^{-1} \leq Q \leq 60 \mathrm{~m}^{3} \mathrm{~s}^{-1}$; les crues quinquennales correspondant à $60 \mathrm{~m}^{3} \mathrm{~s}^{-1} \leq Q \leq 80 \mathrm{~m}^{3} \mathrm{~s}^{-1}$; les crues décennales correspondant à $80 \mathrm{~m}^{3} \mathrm{~s}^{-1} \leq Q \leq 100 \mathrm{~m}^{3} \mathrm{~s}^{-1}$. Ainsi, les forçages de débit du modèle empirique sont le nombre de jours entre deux levés bathymétriques où il y a eu des crues biennales, quinquennales et décennales (figure 2). On remarque une tendance à l'intensification des crues ces dernières années. En effet, on dénombre quatre crues décennales et cinq crues quinquennales depuis 2001 alors que dans les années 90 on dénombre une majorité de crues biennales. Enfin, les dragages réalisés en 1994 $\left(15000 \mathrm{~m}^{3}\right), 2002\left(10000 \mathrm{~m}^{3}\right), 2005\left(15000 \mathrm{~m}^{3}\right)$ et $2007\left(8000 \mathrm{~m}^{3}\right)$ sont pris en comptes dans le modèle via le volume normalisé entre deux levés bathymétriques où il y a eu un dragage.

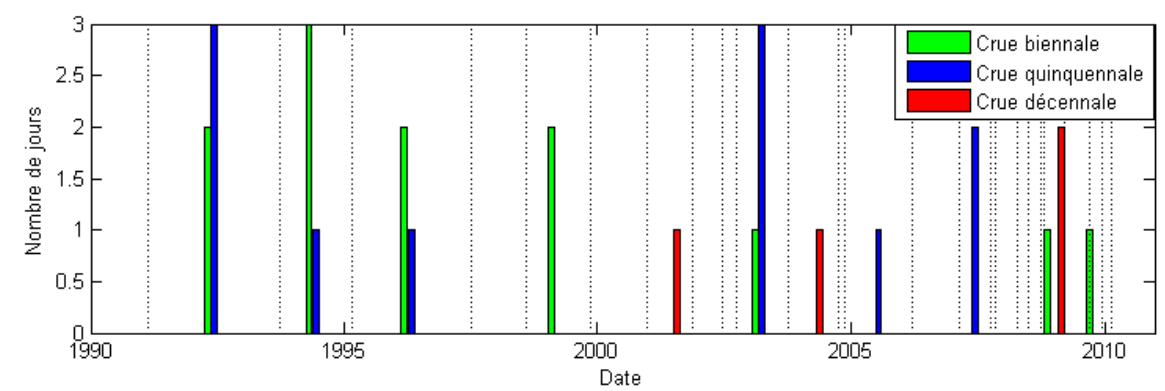

Figure 2. Forçages de débit du modèle et dates des levés bathymétriques (pointillés). 


\section{Mise en ouvre et résultats}

L'analyse factorielle a été mise en œuvre sur 25 levés bathymétriques réalisés entre février 1991 et février 2010. L'AFC requiert que l'emprise des données considérées soit de dimension identique. La zone étudiée est donc limitée aux données communes des 25 levés. Les quatre premières valeurs propres, qui représentent un certain pourcentage de l'inertie des données initiales, montrent que les quatre modes associés expliquent environs 99,6\% de l'inertie des données initiales. Le $1^{\text {er }}$ mode, qui représente la bathymétrie moyenne au cours de la période d'étude, explique à lui seul 97,3\% de l'inertie initiale. Les 3 modes d'ordres supérieurs, qui représentent les fluctuations autour de la bathymétrie moyenne, représentent $84 \%$ de la variance résiduelle. Le $2^{\text {ème }}$ mode prend en compte $50 \%$ de la variance résiduelle et les modes 3 et 4 , qui représentent respectivement $23 \%$ et $12 \%$ de la variance résiduelle ne sont pas étudiés dans la suite de l'étude. Le $1^{\text {er }}$ mode spatial (figure 3.a) représente la bathymétrie moyenne au cours de la période d'étude. Le mode temporel associé $C_{I}(t)$ (figure 3.a) représente les variations de la profondeur moyenne. Les fortes valeurs de $C_{l}(t)$, correspondent à des périodes où la profondeur est plus élevée que la profondeur de la bathymétrie moyenne (cas d'une situation post-dragage). Inversement, les faibles valeurs de $C_{l}(t)$ indiquent des périodes où la profondeur est inférieure à la profondeur de la bathymétrie moyenne (cas d'une situation envasée). Ainsi, le premier mode temporel illustre la tendance globale à l'envasement avec des valeurs de $C_{l}(t)$ plus faibles à partir de 1999 mais également l'augmentation de la fréquence des dragages représentée par les surcroîts rapides de $C_{I}(t)$.
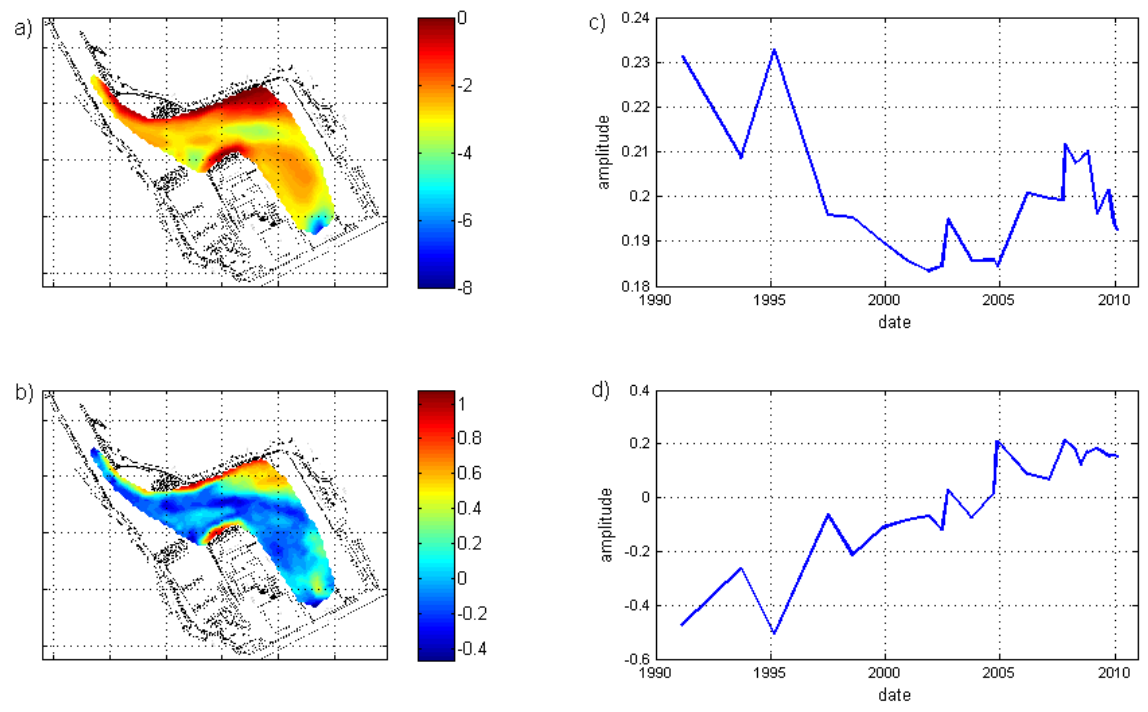

Figure 3. Résultats de l'AFC: $1^{\text {er }}$ mode spatial (a), $1^{\text {er }}$ mode temporel (c), $2^{\text {ème }}$ mode spatial (b), $2^{\text {ème }}$ mode (d). 
Notons que le $1^{\text {er }}$ mode temporel est fortement corrélé $\left(\mathrm{R}^{2}=0,94\right)$ à l'évolution du volume sédimentaire. Le $2^{\text {ème }}$ mode spatial (figure 3.c) permet d'identifier les zones du port qui ont le plus évolué au cours de notre période d'étude. Ainsi, le mode temporel associé $\mathrm{C}_{2}(\mathrm{t})$ (figure 3.d) correspond à un changement de la morphologie du port à l'angle Nord-Est.
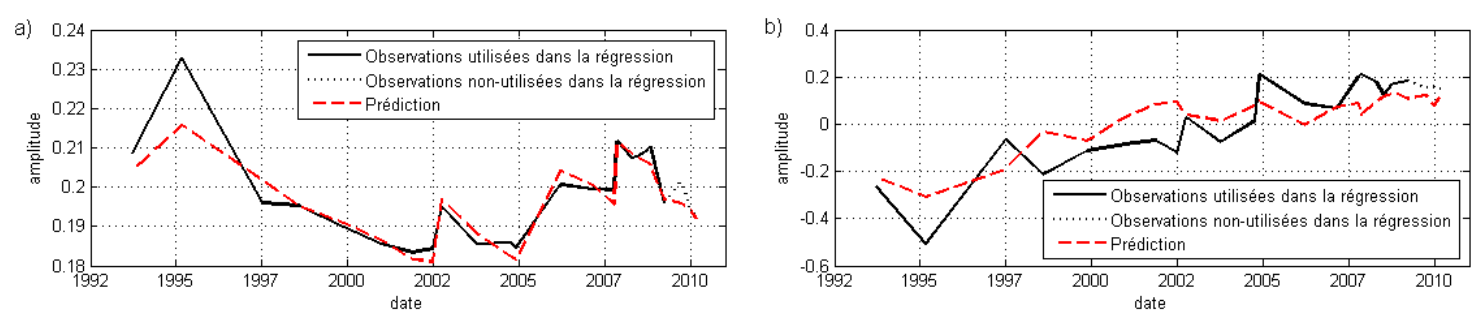

Figure 4. Résultats du modèle linéaire pour les modes 1 (a) et 2 (b).

Les résultats du modèle linéaire pour les deux premiers modes temporels sont illustrés sur la figure 4. Les courbes noires représentent les modes temporels $C(t)$ de l'AFC utilisés dans la régression linéaire, les courbes pointillées noires représentent les modes temporels $C(t)$ de l'AFC non utilisés dans la régression et les courbes en pointillées rouges représentent les modes temporels prédits $\hat{C}(t)$ par le modèle linaire. Le premier mode $\hat{C}_{1}(t)$ obtenu par le modèle reproduit bien l'évolution du premier mode temporel $C_{1}(t)$ issu de l'AFC $\left(\mathrm{R}^{2}=0.93\right)$. Cette bonne corrélation met en évidence la capacité du modèle à reproduire les différents épisodes dragage/envasement répertoriés au cours de la période d'étude. Le deuxième mode $\hat{C}_{2}(t)$ obtenu par le modèle ne reproduit pas toutes les variations de $C_{2}(t)$ issu de l'AFC mais représente correctement la tendance générale $\left(\mathrm{R}^{2}=0.83\right)$ en reproduisant le changement de signe. Ainsi, la forte corrélation entre observations et prédictions du modèle empirique permet de valider les hypothèses réalisées sur les valeurs seuils du débit de la Nivelle et met en évidence le lien entre la tendance renforcée à l'envasement du port et l'augmentation de l'intensité des épisodes de crues depuis 2001.

\section{Conclusions}

Un modèle morphodynamique empirique du port de St-Jean-de-Luz a été développé. L'évolution tridimensionnelle de la bathymétrie entre 1991 et 2010 est prise en compte par AFC et reliée ensuite aux forçages de débit et de dragage via une régression linéaire. Les bons résultats du modèle confirment la pertinence de cette approche et mettent en évidence l'influence de l'intensification des épisodes de crues depuis 2001 sur l'augmentation de la fréquence des dragages. La poursuite d'un suivi bathymétrique régulier devrait permettre d'alimenter le modèle et de renforcer ses capacités prédictives, permettant ainsi au gestionnaire du port de disposer d'un outil de gestion des travaux d'entretient des profondeurs. 
Thème 2 - Dynamique sédimentaire et transports des particules

\section{Références bibliographiques}

AUBREY D.G., INMAN D.L., WINANT C.D. (1980). The statistical prediction of beach changes in southern California. Journal of Geophysical Research, Vol. 85, pp 3246-3276. doi:10.1029/JC085iC06p03264

CHARRITTON X. (1996). Quantification des flux de matières en suspension du ler décembre 1995 au 30 novembre 1996. Etude de l'INRA pour la station d'Hydrobiologie de St Pée sur Nivelle.

COYNEL A., ETCHEBER H., ABRIL G., MANEUX E., DUMAS J., HURTREZ J.E. (2005). Contribution of small mountainous rivers to particulate organic carbon input in the Bay of Biscay. Biogeochemistry, Volume 74, pp 151-171. doi:10.1007/s10533-004-3362-1 CREOCEAN (2001). Port de St-Jean-de-Luz - Notice d'incidence du projet de dragage. Rapport pour le Conseil Général des Pyrénées-Atlantiques.

HSU T.W., LIAW S.R., WANG S.K., OU S.H. (1986). Two dimensional empirical eigenfunction model for the analysis and prediction of beach profile changes. 20th International Conference of Coastal Engineering (Taipei), pp 1180-1195.

HSU T.W., OU S.H., WANG S.K. (1994). On the prediction of beach changes by a new 2-D empirical eigenfunction model. Coastal Engineering, Volume 23, Issues 3-4, pp 255-270. doi:10.1016/0378-3839(94)90005-1

MANEUX E., DUMAS J., CLÉMENT O., ETCHEBER H., CHARRITTON X., ETCHART J., VEYSSY E., RIMMELIN P. (1999). Assesment of suspended matter input into the oceans by small mountainous coastal rivers : the case of the Bay of Biscay. Comptes Rendus de l'Académie des Sciences, Series IIA, Earth and Planetary Science, pp 413-420.

RIHOUEY D. (2004). Analyse statistique de l'évolution morphodynamique des plages sableuses: Application aux sites d'étude du programme national d'environnement côtier et aux plages d'Anglet. Thèse de doctorat de l'Université de Pau et des pays de l'Adour.

RIHOUEY D., DUBRANNA J. (2008). Modèle empirique 3D, Application à la gestion des activités de dragage à l'embouchure de l'Adour. European Journal of Environmental and Civil Engineering, Volume 12, pp 133-146. doi:10.3166/ejece.12.133-146 REEVE D.E., HORRILLO-CARABALlO J.M., MAGAR V. (2008). Statistical analysis and forecasts of long-term sandbank evolution at Great Yarmouth, UK. Estuarine, Coastal and Shelf Science. 79, 3, pp 387-399. doi:10.1016/j.ecss.2008.04.016 SONU C.J., JAMES W.R. (1973). A Markov model for beach profile changes. Journal of Geophysical Research. 78, pp 1462-1471. doi:10.1029/JC078i009p01462 